\title{
Correlation between expression and significance of $\delta$-catenin, CD31, and VEGF of non-small cell lung cancer
}

\author{
X.L. Liu', L.D. Liu' ${ }^{2}$, S.G. Zhang ${ }^{1}$, S.D. Dai ${ }^{3}$, W.Y. Li' ${ }^{1}$ and L. Zhang ${ }^{1}$ \\ ${ }^{1}$ Thoracic Surgery, First Affiliated Hospital, China Medical University, \\ Shenyang, Liaoning, China \\ ${ }^{2}$ Anesthesiology, Affiliated Shengjing Hospital, China Medical University, \\ Shenyang, Liaoning, China \\ ${ }^{3}$ Department of Pathology, First Affiliated Hospital, China Medical University, \\ Shenyang, Liaoning, China \\ Corresponding author: L. Zhang \\ E-mail:zhanglin_xl@163.com \\ Genet. Mol. Res. 14 (4): 13496-13503 (2015) \\ Received March 3, 2015 \\ Accepted June 25, 2015 \\ Published October 28, 2015 \\ DOI http://dx.doi.org/10.4238/2015.October.28.10
}

ABSTRACT. We analyzed the expression and clinical significance of $\delta$-catenin in non-small cell lung cancer (NSCLC) and investigated prognosis using human lung cancer samples. Eighty-nine NSCLC patients underwent operation between January and March 2009. There were 53 cases of squamous cell carcinoma, 31 adenocarcinoma, and 5 large cell carcinoma. $\delta$-Catenin in NSCLC patients was detected by immunohistochemistry and analyzed in combination with the clinicopathological characteristics of lung cancer. The relationship between $\delta$-catenin and CD31, D2-40, and vascular endothelial growth factor (VEGF) was compared by immunohistochemistry and the $\mathrm{X}^{2}$ test. $\delta$-Catenin appeared in the cytoplasm of adjacent bronchial epithelial cells, indicating negative expression. Positive $\delta$-catenin expression in the cytoplasm of lung cancer tissues was $66.67 \%$ (52/78), which was significantly higher than in normal lung tissues. Kaplan-Meier survival analysis suggested that the mean survival time of patients with $\delta$-catenin-positive expression was significantly shorter than in those 
with negative expression, indicating that positive expression was closely related to poor prognosis of NSCLC. $\delta$-Catenin was highly expressed in NSCLC mainly in the cytoplasm of lung cancer tissues. $\delta$-Catenin-positive expression may be related to poor prognosis of NSCLC. High $\delta$-catenin expression in NSCLC was positively correlated with high CD31 and VEGF expression, but not correlated with D2-40, suggesting that $\delta$-catenin may be related to angiogenesis and not lymphangiogenesis.

Key words: Angiogenesis; CD31; ס-Catenin; D2-40; Non-small cell lung cancer; Vascular endothelial growth factor

\section{INTRODUCTION}

Lung cancer is a malignant tumor and currently has the highest incidence rate worldwide, accounting for approximately $12 \%$ of total cancer cases. Non-small cell lung cancer (NSCLC) accounts for $80 \%$ of all lung cancers (Ren et al., 2004). Migration of cancer is the primary cause of death of patients with NSCLC, while neovascularization plays an important role in tumor growth and migration (Folkman, 1990) and provides metabolic nutrients for metastatic tumors (Zhao et al., 2011; Bowden and Barrett, 2011). Therefore, it is important to understand the mechanisms of angiogenesis in lung cancer to decrease mortality rate and determine patient prognosis.

$\delta$-Catenin (neural plakophilin-related armadillo protein) is an important member of the p120ctn family. Recent studies have revealed that $\delta$-catenin is highly expressed in various tumors and plays a prominent role in tumor invasion and migration. However, there have been few reports regarding the relationship between this protein and neovascularization of NSCLC. Numerous studies have demonstrated that tumor neovascularization is an extremely complex process involving the interaction of a variety of cytokines. CD31 is the main marker of neovasculature endothelial cell proliferation, and thus labels endothelial cells with proliferative activity. CD31 is mainly expressed in neovasculature endothelial cells of tumors and is a highly sensitive marker. The vascular endothelial growth factor (VEGF) receptor signaling pathway participates in the migration, proliferation, and survival of new endothelium cells adjacent to tumors and plays a critical role in the neonatal process of neovasculature adjacent to tumors. A meta-analysis by Deimotte et al. (2002) found that VEGF is a marker for the prognosis of NSCLC patients. In this study, the expression of $\delta$-catenin in 78 cases NSCLC patients was detected using immunohistochemistry. Two critical markers, CD31 and VEGF, were used to investigate the relationship between $\delta$-catenin and neovascularization of lung cancer.

\section{MATERIAL AND METHODS}

\section{Samples and subjects}

Eighty-nine patients with NSCLC underwent operation in the Thoracic Surgery Department of the First Affiliated Hospital of China Medical University from January to March 2009 and were included in the study, comprising 53 men and 36 women, aged 35-78 years (mean 54.7 years). Histology was classified according to the WHO histological standard in 2004 and included 53 cases squamous cell carcinoma, 31 cases adenocarcinoma, and 5 cases large cell carcinoma. Patients were judged according to the 7th edition of TNM staging criteria by Union International Control Cancer in 2009: 39 cases of stage I, 25 cases of stage II, and 25 cases of stage III; 78 cases had detailed postoperative 
follow-up data. The survival times of patients were calculated as operation date to death date caused by recurrence and metastasis or to final follow-up date (March 2014). Additionally, we collected 10 cases of lung cancer and normal tissue adjacent to the cancer and analyzed these samples by immunohistochemistry. The protocol was approved by the Ethical Review Committees of the hospital. Written informed consent to participate in the study was obtained from each patient.

\section{Methods}

Lung tissues were fixed with $40 \mathrm{~g} / \mathrm{L}$ formalin, embedded in paraffin, and sliced into $4 \mathrm{~mm}$ sections. Aquaporin-1 protein was labeled using the immunohistochemistry S-P method. Slices were stained with 3,3'-diaminobenzidine and counter-stained with hematoxylin before mounting. In the control group, primary antibodies were replaced with phosphate-buffered saline. The results were observed under a microscope.

\section{Statistical analysis}

Statistical analysis was performed using the SPSS version 19.0 software (SPSS, Inc., Chicago, IL, USA). $X^{2}$ analysis was used to analyze the relationship between $\delta$-catenin and the expression of CD31 and VEGF. Kaplan-Meier analysis was used to analyze patient prognosis. Significance analysis was conducted using the log-rank method. $\mathrm{P} \leq 0.05$ was considered to be statistically significant.

\section{RESULTS}

\section{High expression of $\delta$-catenin in lung cancer tissues}

Positive staining of $\delta$-catenin expression showed various light yellow and grey particles in the cytoplasm. There was light signal in the cytoplasm of adjacent bronchial epithelial cells and glandular epithelia cells (Figure 1A), which was determined to be negative expression according to the grading standard. The positive $\delta$-catenin signal was located in the cytoplasm of lung cancer tissues (Figure 1B and C), showing significantly enhanced expression. The positive expression rate was $66.67 \%(52 / 78)$, which was significantly higher than in expression in normal lung tissues (Figure 2A-F and 3A-F).
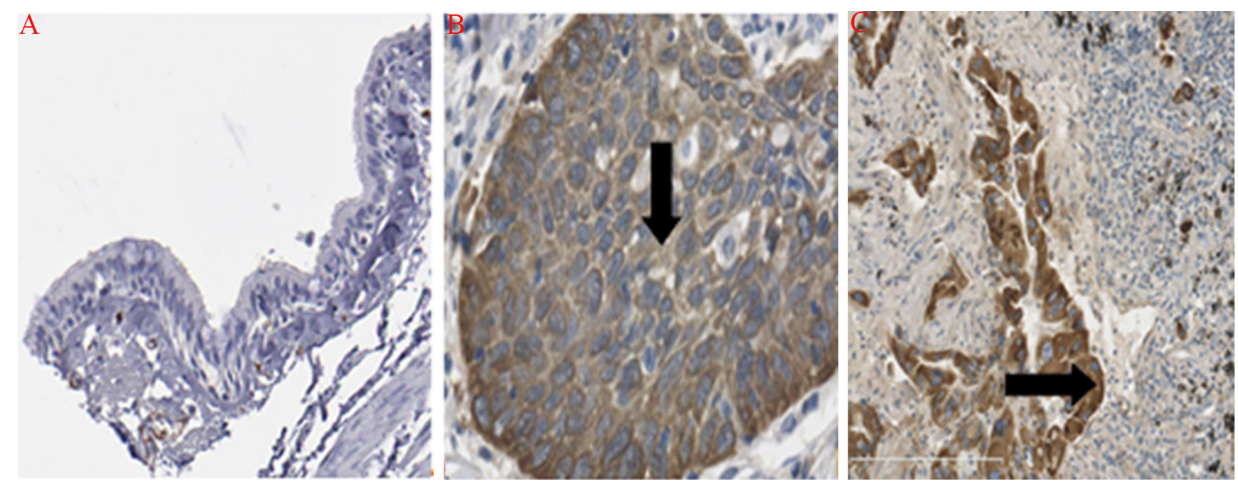

Figure 1. Immunohistochemical staining result of $\delta$-catenin in adjacent normal lung tissues (A), squamous cell carcinoma (B), and adenocarcinoma (C) (A. 200X; B. C. 400X). 

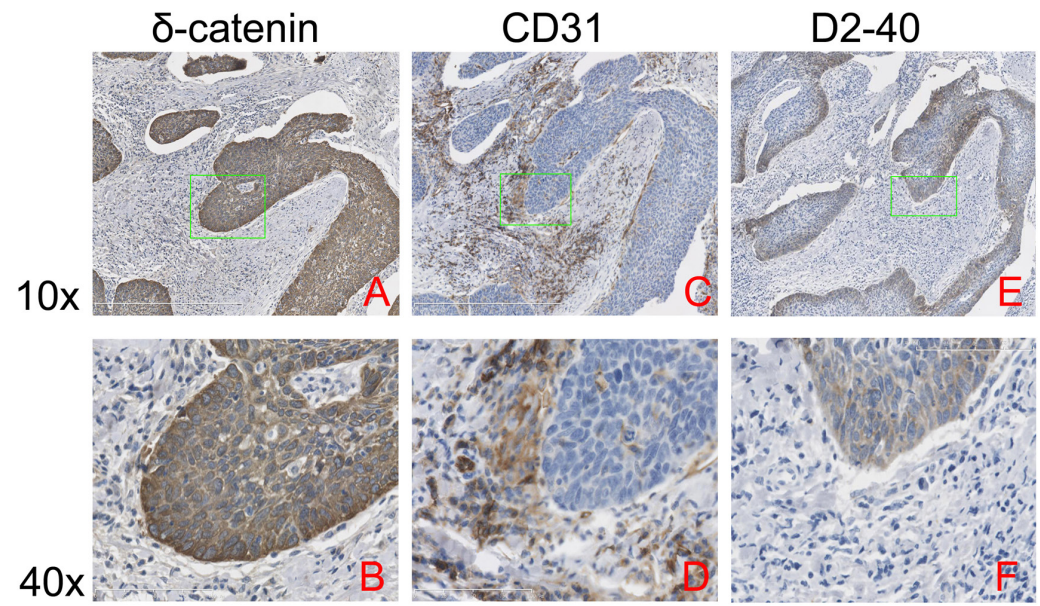

Figure 2. Immunohistochemical staining result of $\delta$-catenin, CD34, and D2-40 in lung squamous cell carcinoma (A. C. E. 100X; B. D. F. 400X).
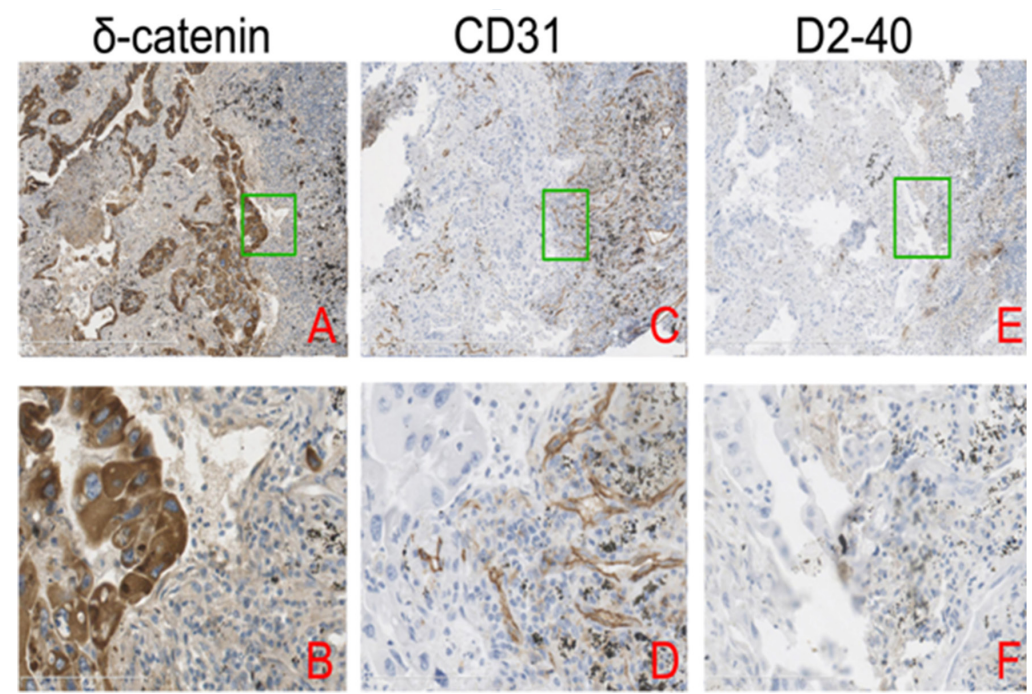

Figure 3. Immunohistochemical staining result of $\delta$-catenin, CD34, and D2-40 in lung adenocarcinoma (A. C. E. 200X; B. D. F. 400X).

\section{Relationship between $\delta$-catenin and clinicopathological features and prognosis of lung cancer patients}

The positive expression rate of $\delta$-catenin was $66.67 \%$ (52/78) and significantly higher than the positive expression rate in adjacent bronchial epithelial cells. The positive expression rate of $\delta$-catenin showed no significant relationship with gender, age, and differentiation grades (Table 1, $P>0.05$ ), while expression was related to histological type and TNM period. The positive expression rate of lung cancer was $79.5 \%$, which was significantly higher than the $57.1 \%$ observed 
in squamous cell carcinoma $(P<0.05)$. The positive expression rate in stage III cases was $90.9 \%$, which was significantly higher than the $47.4 \%$ observed in stage I and $56.8 \%$ observed in stage II $(P<0.05)$ patients. The 5-year survival rate of the 78 cases lung cancers with detailed postoperative follow-up data was $39.7 \%$ in patients with $\delta$-catenin-positive expression and $53.8 \%$ in those with $\delta$-catenin-negative expression. The results of single factor analysis revealed that $\delta$-catenin-positive expression was closely related to the poor prognosis of lung cancers $(P<0.05$, log-rank method, Figure 4).

The average survival time and survival rate 5 years after operation was significantly lower in the lung cancer cases showing $\delta$-catenin-positive expression (blue) compared to in $\delta$-cateninnegative expression (green) $(P<0.05)$.

Table 1. Relationship between $\delta$-catenin and clinicopathological features of lung cancers.

\begin{tabular}{|c|c|c|c|c|c|}
\hline \multirow[t]{2}{*}{ Group } & \multirow[t]{2}{*}{ Cases } & \multicolumn{4}{|c|}{$\delta$-Catenin } \\
\hline & & Positive cases & $\%$ & $x^{2}$ & $P$ \\
\hline Age (years) & & & & 0.731 & $>0.05$ \\
\hline$<60$ & 35 & 23 & 65.7 & & \\
\hline$\geq 60$ & 43 & 29 & 67.4 & & \\
\hline Gender & & & & 0.0008 & $>0.05$ \\
\hline Male & 51 & 31 & 60.8 & & \\
\hline Female & 27 & 21 & 70.8 & & \\
\hline TNM period & & & & 6.412 & $<0.05$ \\
\hline 1 & 19 & 9 & 47.4 & & \\
\hline II & 37 & 21 & 56.8 & & \\
\hline III & 22 & 20 & 90.9 & & \\
\hline Tissue type & & & & 6.549 & $<0.05$ \\
\hline Adenocarcinoma & 39 & 31 & 79.5 & & \\
\hline Squamous cell carcinoma & 35 & 20 & 57.1 & & \\
\hline Large cell carcinoma & 4 & 1 & 20 & & \\
\hline
\end{tabular}

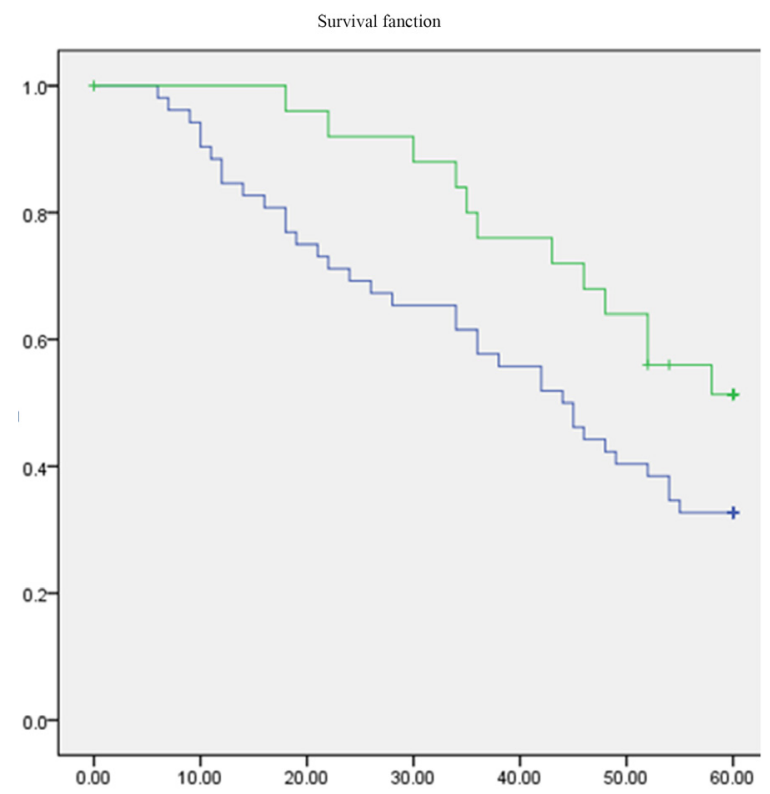

Figure 4. Kaplan-Meier survival curve of lung cancer with different expression models of $\delta$-catenin. 


\section{$\delta$-Catenin expression was positively correlated with CD31 expression}

As shown in the Table 2, high expression of $\delta$-catenin in NSCLC was positively correlated with high expression of $\operatorname{CD} 31\left(X^{2}=16.7, P<0.01\right)$.

\begin{tabular}{|c|c|c|c|}
\hline \multirow[t]{2}{*}{ CD31 } & \multicolumn{2}{|c|}{$\delta$-Catenin } & \multirow[t]{2}{*}{ Total } \\
\hline & + & - & \\
\hline+ & 42 & 8 & 50 \\
\hline - & 10 & 18 & 28 \\
\hline Total & 52 & 26 & 78 \\
\hline$x^{2}$ & 16.7 & & \\
\hline
\end{tabular}

\section{ס-Catenin expression was not related to D2-40 expression}

As shown in Table 3, high expression of $\delta$-catenin in NSCLC was not related to the high expression of D2-40 $\left(X^{2}=0.317, P>0.05\right)$.

\begin{tabular}{lccc}
\multicolumn{4}{c}{ Table 3. Relationship between $\delta$-catenin and the expression of D2-40. } \\
\hline D2-40 & D-Catenin & Total \\
\cline { 2 - 4 } & + & 10 & 25 \\
\hline+ & 25 & 16 & 42 \\
- & 27 & 26 & 78 \\
Total & 52 & & \\
$X^{2}$ & 0.317 & & \\
\hline
\end{tabular}

\section{Positive correlation between $\delta$-catenin expression and VEGF expression}

As shown in Table 4, there was a significantly positive correlation between high expression of $\delta$-catenin in NSCLC and high expression of VEGF $\left(X^{2}=7.74, P<0.05\right)$ (Figure $5 A$ and $B$ ).

\begin{tabular}{|c|c|c|c|}
\hline \multirow[t]{2}{*}{ VEGF } & \multicolumn{2}{|c|}{$\delta$-Catenin } & \multirow[t]{2}{*}{ Total } \\
\hline & + & - & \\
\hline+ & 40 & 7 & 47 \\
\hline- & 12 & 19 & 31 \\
\hline Total & 52 & 26 & 78 \\
\hline$x^{2}$ & 7.74 & & \\
\hline
\end{tabular}

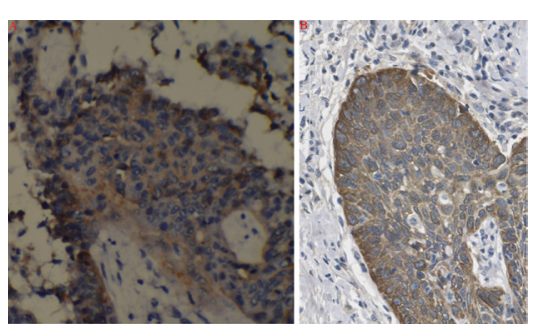

Figure 5. Immunohistochemical staining result of $\delta$-catenin and VEGF in lung cancer. A. High expression of VEGF (200X). B. High expression of $\delta$-catenin (200XX). 


\section{DISCUSSION}

$\delta$-Catenin is an important member of the p120ctn family, the members of which are highly expressed in the central nervous system of normal people (Kosik et al., 2005). Recently, the role of $\delta$-catenin was studied in various tumors (Zhang et al., 2010) and was found to be highly expressed in a variety of malignant tumors. Consistent with other studies (Dai et al., 2011), we found that high $\delta$-catenin expression in NSCLC was related to poor prognosis. The positive expression rate of $\delta$-catenin was $66.67 \%$ (52/78) in this study, which was significantly higher than the expression in normal lung tissues. The survival curve showed that prognosis was poorer in patients with $\delta$-cateninpositive expression. Numerous factors influence lung cancer prognosis and many related genes are involved. Angiogenesis and lymphangiogenesis are important in tumor incidence, invasion, and metastasis. We conducted a preliminary investigation to examine the relationship between $\delta$-catenin and angiogenesis and lymphangiogenesis by determining the relationship between the expression of $\delta$-catenin vs CD31 and D2-40 in the NSCLC.

The monoclonal antibody D2-40 is highly specific and sensitive in lymphatic endothelial cells (Takanami, 2006) and shows a negative reaction in vascular endothelial cells. It is considered to the specialist marker for newborn lymphatic endothelial cells (Fukunaga, 2005). A previous study showed that lymphangiogenesis can be accurately assessed in tumors by using D2-40 to label lymphatic endothelial cells in NSCLC (Hashizume et al., 2009). Immunohistochemical results in lung cancer tissues revealed that $\delta$-catenin expression was not related to D2-40 expression, suggesting that no relationship exists between $\delta$-catenin and NSCLC prognosis and lymphangiogenesis.

CD31 is platelet endothelial cell adhesion molecule expressed by the endothelium of both lymphatic and blood vessels as well as an endothelium marker. CD31 is mainly distributed in pulse tube cells; it is closely related to angiogenesis and the locomotion of endothelial cells. Additionally, CD31 acts as a bridge linking tumor cells and vascular endothelial cells and promotes the migration of malignant tumors (Raspollini et al., 2004). CD31 is a commonly used vascular marker in the clinic, and is an angiogenesis regulatory factor in the immunoglobulin superfamily, for which the antibody specifically labels vascular endothelial cells. The results of this study showed that positive expression of $\delta$-catenin was significantly related to overexpression of CD31, suggesting that the influence of $\delta$-catenin in NSCLC patient prognosis may be related to angiogenesis; however, the mechanism of how $\delta$-catenin influences angiogenesis in NSCLC remains unclear.

VEGF is very important among the various cytokines induced during angiogenesis (Guo et al., 2011). VEGF plays a role in strongly inducing the expression of its receptor. This can improve vascular permeability through its special receptor, which is beneficial for extravasation of plasma proteins as well as to promote the amplification of vascular endothelial cells specifically (Ferrana, 1999). VEGF and its receptor jointly control the differentiation of endothelial cells and angiogenesis through the autocrine and paracrine systems. Additionally, they play key roles in promoting division and proliferation of endothelial cells as well as angiogenesis. In this study, the expression of VEGF was high in NSCLC patients and significantly related to the positive expression of $\delta$-catenin. That indicates that $\delta$-catenin cooperates with VEGF to promote angiogenesis in NSCLC. Further studies are needed to determine the relationship between $\delta$-catenin and angiogenesis in NSCLC as well as the related mechanism.

\section{Conflicts of interest}

The authors declare no conflict of interest. 


\section{ACKNOWLEDGMENTS}

Research the supported by National Natural Science Foundation (\#81201890).

\section{REFERENCES}

Bowden DJ and Barrett J (2011). Angiogenesis imaging in neoplasia. J. Clin. Imaging Sci. 1: 38.

Dai SD, Wang Y, Zhang TY, Zhang D, et al. (2011). Upregulation of $\delta$-catenin is associated with poor prognosis and enhances transcriptional activity through Kaiso in non-small-cell lung cancer. Cancer Sci. 102: 95-103.

Deimotte P, Martin B, Pasemans M, Berghmans T, et al. (2002). VEGF and survival of patients with lung cancer: a systematic literature review and meta-analysis. Rev. Mal. Respir. 19: 577-584.

Ferrana N (1999). Molecular and biological properties of vascular endothelial growth factor. J. Mol. Med. 77: 527-543.

Folkman J (1990). What is the evidence that tumors are angiogenesis dependent? J. Natl. Cancer Inst. 3: 4-6.

Fukunaga M (2005). Expression of D2-40 in lymphatic endothelium of normal tissues and in vascular tumours. Histopathology 46: 396-402.

Guo P, Xu L, Pan S, Brekken RA, et al. (2011). Vascular endothelial growth factor isoforms display distinct activities is promoting tumor angiogenesis at different anatomic sites. Cancer Res. 61: 8569-8577.

Hashizume S, Nagayasu T, Hayashi T, Hidaka S, et al. (2009). Accuracy and prognostic impact of a vessel invasion grading system for stage IA non-small cell lung cancer. Lung Cancer 65: 363-370.

Kosik KS, Donahue CP, Israely I, Liu X, et al. (2005). Delta-catenin at the synaptic-adherens junction. Trends Cell. Biol. 15: 172-178.

Raspollini MR, Amunni G, Villanucci A, Baroni G, et al. (2004). Prognostic significance of microvessel density and vascular endothelial growth factor expression in advanced serous carcinoma. Int. J. Gynecol. Cancer 14: 815-823.

Ren H, Tang X, Lee JJ, Feng L, et al. (2004). Expression of hepatoma-derived growth factor is a strong prognostic predictor for patients with early-stage non-small-cell lung cancer. J. Clin. Oncol. 22: 3230-3237.

Takanami I (2006). Lymphatic microvessel density using D2-40 is associated with nodal metastasis in non-small-cell lung cancer. Oncol. Rep. 15: 437-442.

Zhang JY, Wang Y, Zhang D, Yang ZQ, et al. (2010). delta-Catenin promotes malignant phenotype of non-small cell lung cancer by non-competitive binding to E-cadherin with P120ctn in cytoplasm. J. Pathol. 222: 76-88.

Zhao C, Wang X, Zhao Y, Li Z, et al. (2011). A novel xenograft model in zebrafish for high-resolution investing dynamics of neovascularization in tumors. PLoS One 6: e21768. 